\title{
AN ANALYSIS OF CONTEMPORARY METHODS FOR MEASUREMENT OF FORM ERRORS OF SPHERICAL MACHINE PARTS
}

\author{
Stanisław Adamczak, Dariusz Janecki, Krzysztof Stępień, Mateusz Wrzochal
}
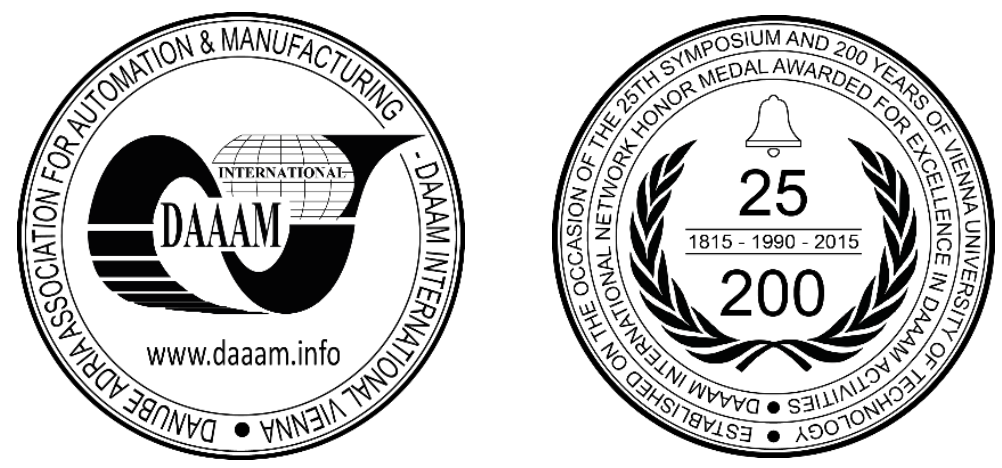

This Publication has to be referred as: Adamczak, S[tanislaw]; Janecki, D[arius]; Stepien, K[rzysztof] S[tanislaw] \& Wrzochal, M[ateusz] (2017). An analysis Of Contemporary Methods For Measurement Of Form Errors Of Spherical Machine Parts, Proceedings of the 28th DAAAM International Symposium, pp.1101-1107, B. Katalinic (Ed.), Published by DAAAM International, ISBN 978-3-902734-11-2, ISSN 1726-9679, Vienna, Austria

DOI: $10.2507 / 28$ th.daaam.proceedings. 153

\begin{abstract}
Spherical elements constitute significant group of machine parts. In particular, they are commonly applied in bearing industry. Spherical elements should be usually manufactured very precisely and their form deviations are relatively small. Deviations of balls used in rolling bearings usually do not exceed 1 micrometer. The problem of accurate measurement of spherical parts is complicated and standards do not provide too many recommendations referring to this problem. The paper presents up-to-date solutions of the problem of measurements of spherical parts. It also presents the method of sphericity measurements developed at Kielce University of Technology. In addition, measurement strategies are presented that are based on developed method.
\end{abstract}

Keywords: measurement; methods; sphericity; form deviations; measurement systems

\section{Introduction}

A key issue in contemporary manufacturing technology is to control dimensional accuracy of machine elements. Apart from the dimensions, quality of surfaces of machine parts is very important, too. This is why accurate surface measurements are nowadays a matter of great importance [1-3]. Considering measurements of rotary elements it is noteworthy that problems of measurements of roundness and cylindricity are well described in the scientific literature.

However, measurements of roundness seem to be insufficient way to evaluate form deviations of spherical elements. This is why in a number of research centres studies focused on this problem are conducted.

The main aim of this study is to critically evaluate major advantages and drawbacks of sphericity measurement methods that are presented in the scientific literature. Additionally, the concept of sphericity measurement developed by authors is presented. The concept is based on applying the radius-change instrument equipped with special-purpose unit for controlled rotation of spheres 


\section{Sphericity measurements by the contact method}

Generally methods of measurements of sphericity can be divided into two main groups: contact and optical methods. Contact methods can be classified as industrial and laboratory ones. Industrial methods apply simple instruments and provide quick information if measured element fulfils its technological requirements. Laboratory methods are more complex and they aim at as accurate measurement of the whole surface of the specimen as possible. In the laboratory methods the process of measurement is usually much longer and complicated than in the industrial methods.

\subsection{Industrial methods}

Standardization documents used in the industrial practice describe following strategy of measurements of sphericity of machine elements: roundness profiles to be measured in three mutually perpendicular cross-sections of the specimen (see Fig. 1). Results of the measurements are presented in the form of polar diagrams and the diagrams are the basis to evaluate approximate sphericity deviations. Such approach allows rough evaluation of sphericity only, as significant part of the surface is not sampled. Moreover, it should be noted that in industrial practice evaluation of form deviations of spherical elements is conducted on the basis of an analysis of only one roundness profile measured in a random crosssection of the sphere.

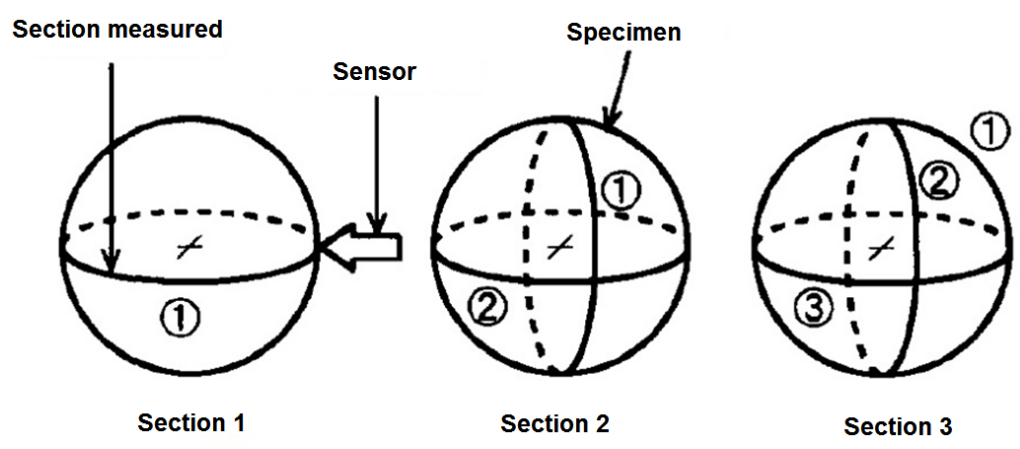

Fig. 1. Strategy of measurements of spherical elements described in industrial standards [4]

Industrial documents state usually that the best reference feature to evaluate form deviations of spherical elements is the least circle circumscribed on the measured profile. It is noteworthy that industrial approach does not require to apply any special-purpose systems for sphericity measurements, but recommends using a typical roundness measurement instruments. Usually such instruments are based on the radius-change method.

\subsection{Laboratory measurements}

Apart from the radius-change method also a 3-point method can be used to measure sphericity, which was described in [5]. Generally, in this concept the sphere is placed on two-point support [see Fig. 2]. The signal is obtained from the sensor whose axis intersects the nominal centre of the sphere. During the measurement the sphere rotates and due to irregularities of the surface the sensor readings change. This concept requires mathematical transformation of the sensor readings since the signal is affected by irregularities occurring in all three contact points of the sphere. It can be easily conducted with the use of Fourier transform [6, 7].

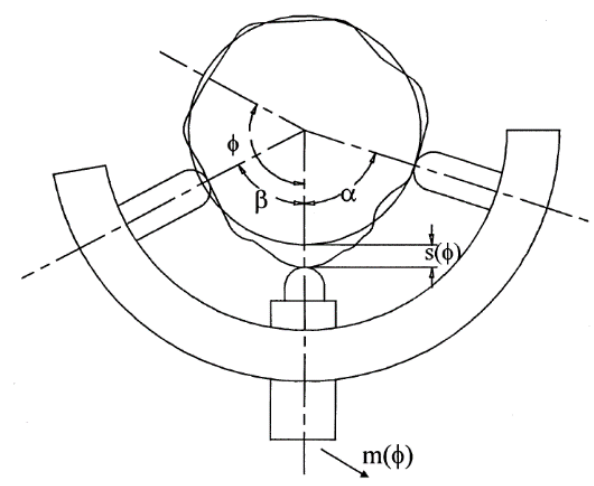

Fig. 2. Sphericity measurements by the 3 -point method . $\varphi$ - rotation angle, $\mathrm{s}(\varphi)$ - deviation, $\mathrm{m}(\varphi)-\operatorname{sensor}$ readings, $\alpha, \beta$ angles between supports and the sensor axis [5] 
Sensor readings in a given point of the profile is a function of the angles between supports and the change of the displacement of the sensor tip in this point. The major drawback of this method is that there are some harmonic components of the profile that cannot be detected by the measuring system.

Other concept of sphericity measurements has been developed at Kielce University of Technology [8]. This concept is based on using typical roundness measuring instrument additionally equipped with e special-purpose unit allowing accurate rotation of measured spheres (see Fig. 3). Owing to that it is possible to measure roundness profiles of the sphere in a number of strictly defined cross-sections.

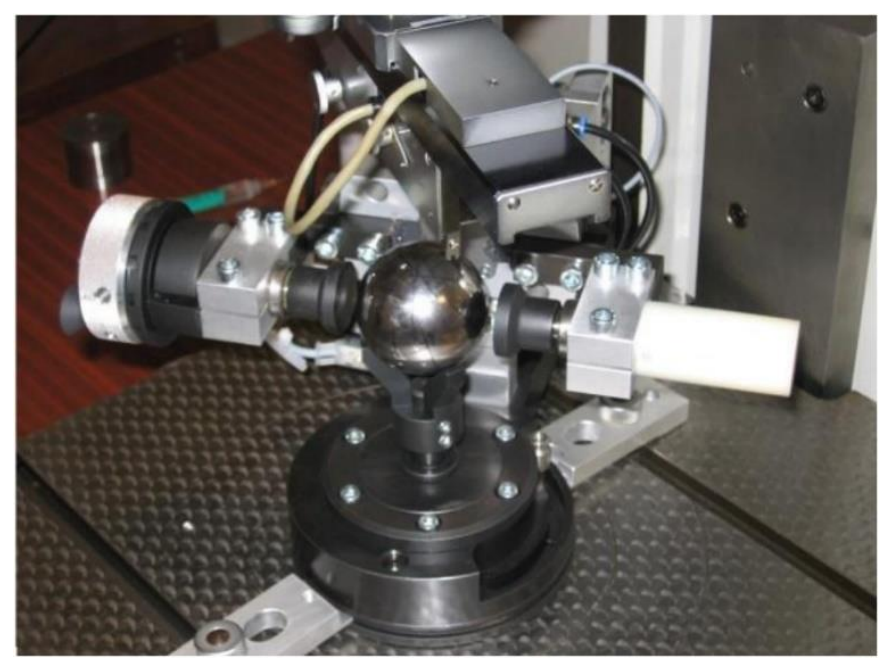

Fig. 3. The unit for accurate positioning of spheres

\section{Non-contact methods of sphericity measurements}

Except for contact methods, a number of researchers focus on studying methods of sphericity measurements with the use of optical systems. Such methods can be divided into two main groups: measurements applying the light beam and measurements based on image processing.

\subsection{Light beam measurements}

It should be noted that already in the sixties of the XX. century in former Czechoslovakia an automated system for quality control of balls of rolling bearing has been developed that applied an optical system. The system operated on the basis of observing changes of luminosity of the light reflected from the sphere surface. The spheres during measurements were rotated by the rolls. Focused light beam falls on the surface of the sphere and the reflects to a photodiode. If the surface is damaged then the luminosity of the light reflected changes [9].

At present, intensive research work is carried out in the field of measurements of surface topography of spherical elements with the use of laser interferometers, which was described for example in work [10]. In the method presented in Fig. 4 small fragments of the sphere are measured with the use of Fizeau interferometer and then the procedure of stitching is applied to obtain the image of the whole surface.

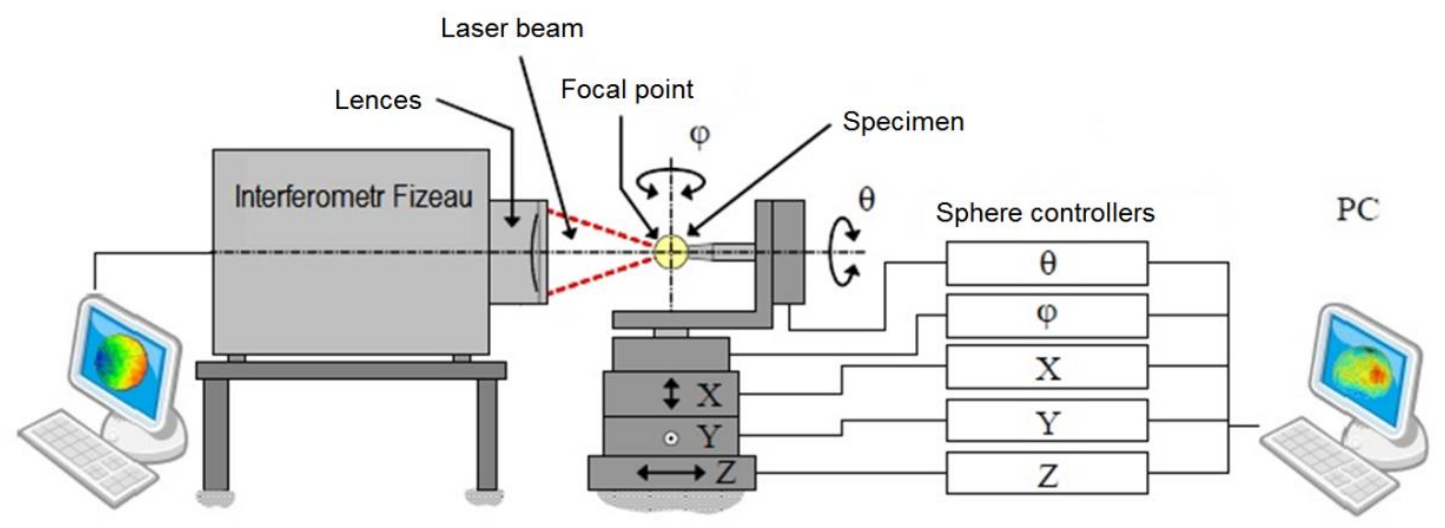

Fig. 4. Measurements of sphericity by Fizeau interferometer presented in work [10] 
Method presented in Fig. 4 requires to determine accurate position of the sphere during subsequent measurements. If the position of the sphere were not determined accurately then it would not be possible to stitch the fragments. Thus, rotation of the sphere is performed with a set of controllers. Relatively similar system is described in work [11]. This system applies a modified Fizeau interferometer, too. Similarly to the method presented in [11] the image of the whole sphere is reconstructed from measured fragments, which is shown in Fig. 5.

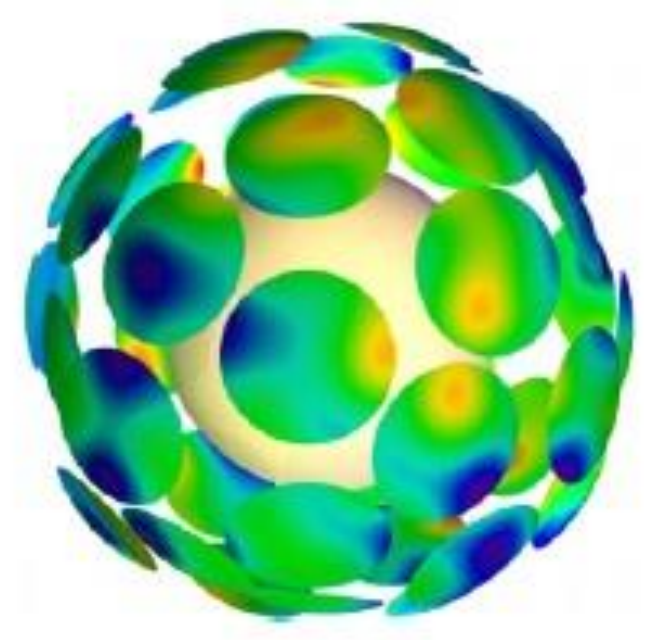

Fig. 5. Reconstruction of the image of the sphere from measured fragments of the surface [11]

\subsection{Measurements applying image processing methods}

In work [12] the method for determination of sphericity deviation of the electrodischarge machining tool with the use of an image processing techniques. The instrument is here properly calibrated camera that observes an image of the tool tip. Next, relevant software is used to determine coordinates of points lying on the edge of the tool, which is shown in Fig. 6.

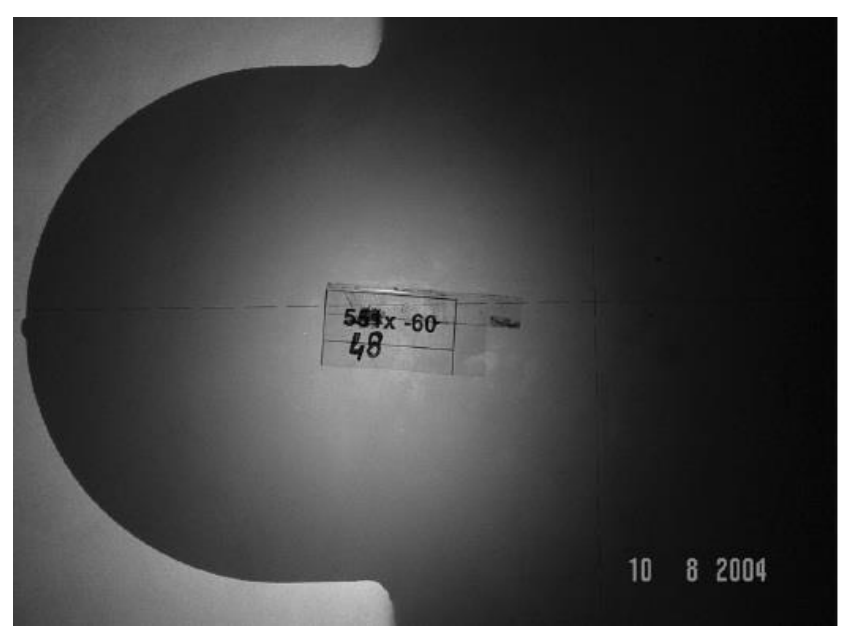

Fig. 6. One of the images used to evaluate sphericity deviations of the tool [10]

After determining the coordinates in a certain position the specimen is rotated to the next position and the procedure repeats then. This way a matrix containing values of coordinates of the sphere is obtained. The values from the matrix are then used to evaluate relevant roundness and sphericity parameters.

An interesting method allowing sphericity measurements by the optical method is presented in work [13]. Authors of this work propose to apply digital image processing technique to reconstruct a 3D view of the surface from twodimensional images. In the presented method a semisphere can be reconstructed from 2D images. The method applies properly calibrated camera to get 2D images. As distances of various points on the sphere are different then the intensity of shading scale is different, too. The changes of this intensity is the basis to generate 3D view of the surface. One of the 2D images and reconstructed 3D view of the surface are shown in Fig. 7. 
a)

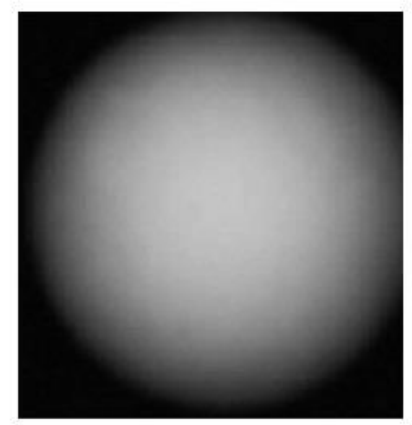

b)

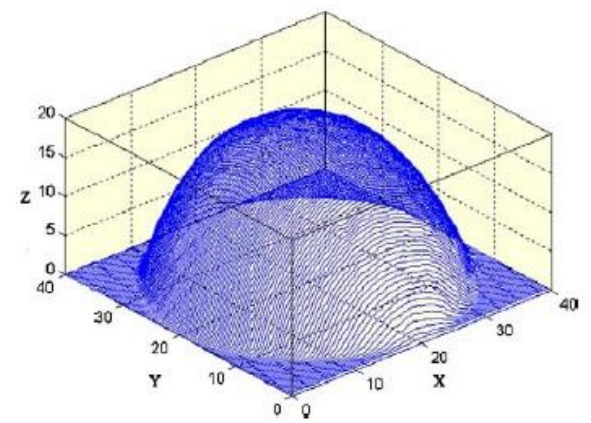

Fig. 7. Reconstruction of the $3 \mathrm{D}$ view of the surface from 2D images of the sphere: a) an image captured by the camera. b) reconstructed 3D view [13]

\section{Sphericity measurement strategy in contact measurements}

At present most common instruments used to measure form deviations of rotary elements are radius-change systems, described for example in work [14]. Equipping such systems with a unit for accurate positioning of the spheres, shown in Fig. 3 allows application of various measurement strategies that permit covering the sphere with a dense grid of sampling points. Authors have conducted a series of preliminary measurements to find most useful strategies that could be used to evaluate form deviations of spherical elements. Two of these strategies are shown in Fig. 8 and Fig. 9. Fig. 8 shows a socalled equator\&meridians strategy.

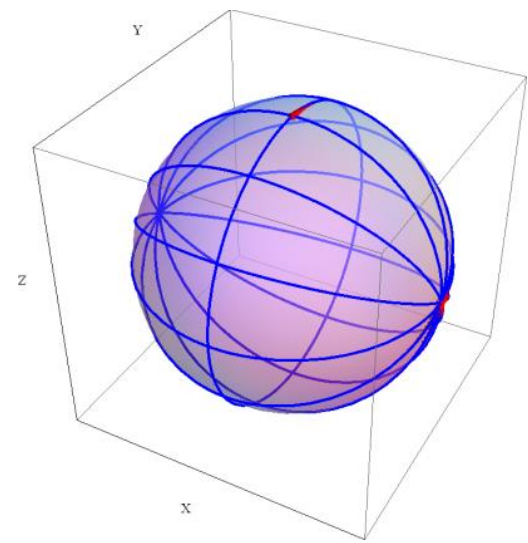

a)

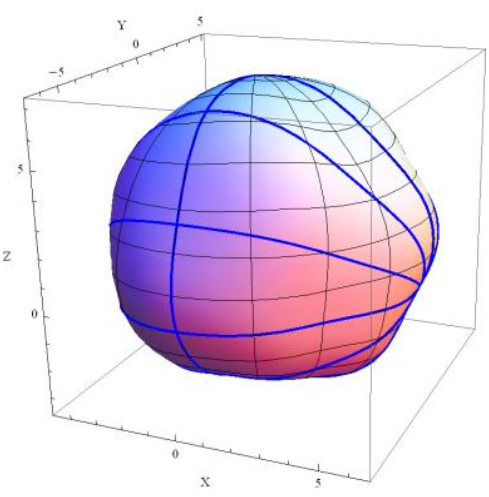

b)

Fig. 8. Equator\&meridians strategy: a) scanning trajectory, b) distribution of measured sections on a real element Another analyzed strategy was a "bird-cage" strategy shown in Fig. 9.

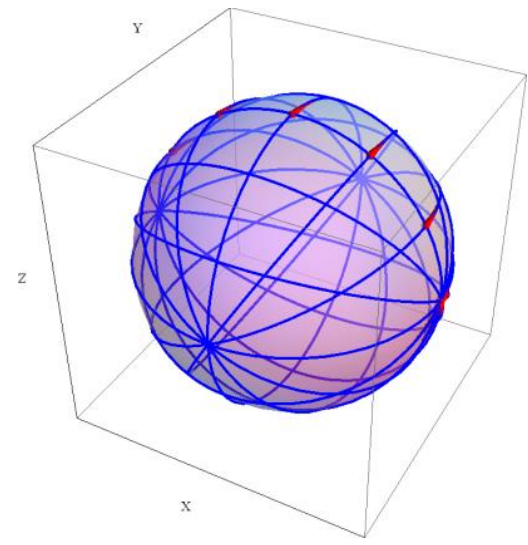

a)

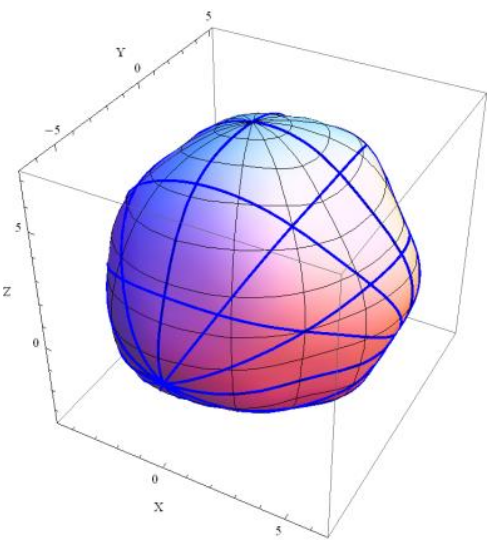

b)

Fig. 9. "Bird-cage" strategy: a) scanning trajectory, b) distribution of measured sections on a real element. 
The unit shown in Fig. 3 allows applying many more measurement strategies that are more complicated that those presented above. However, experience of authors indicates that in practice "bird-cage strategy" provides high quality results $[15,16]$. It should be noted that accurate evaluation of sphericity deviations with such strategy requires performing a number of mathematical operations used to process measurement data, such as: profiles matching, filtration, etc. [1719].

\section{Conclusions}

The problem of accurate measurements of sphericity devations is very complex. In industrial practice contact methods are dominant, however an industrial approach to evaluate form errors of spheres is very simplified. A number of researchers deals also with studying non-contact methods to measure out-of-sphericity. Apart from that coordinate measuring machines are investigated to apply in such measurements. Considering dynamic development of coordinate metrology it is possible that in the near future this technique will be one of the leading when measuring spherical elements. However, at present time, coordinate measuring machine do not still offer accuracy sufficient to satisfy manufacturers of precise spheres used for example in bearing industry.

Generally, it is noteworthy that laboratory methods presented in sections 2.2 and 3 are quite complicated from practical point of view, as they usually require complicated measurement data processing procedures. This is why it would be very difficult to apply them under industrial conditions. The solution can be the method developed by authors which is based on applying a typical radius-change measuring instrument equipped with the unit for controlled rotation of spheres. Owing to such system it is possible to conduct a series of measurements of roundness profiles that can be further used to reconstruct a $3 \mathrm{D}$ view of the surface of measured element.

Additionally, it must be underlined that no matter what method is used to measure spherical parts, accurate evaluation of form errors of sphericity deviations is generally very complex and difficult. It requires applying complicated mathematical apparatus using spherical harmonic components and complex measurement data procedures.

\section{Acknowledgment}

The paper has been elaborated within the framework of the research project entitled "Theoretical and experimental problems of integrated 3D measurements of elements' surfaces", reg. no.: 2015/19/B/ST8/02643, ID: 317012, financed by National Science Centre, Poland.

\section{References}

[1] Jermak C. J., Rucki M. (2016), Static Characteristics of Air Gauges Applied in the Roundness Assessment, Metrology and Measurement Systems, 23 (1), 85-96.

[2] Zorawski W. et al. (2015), Microstructure and tribological properties of nanostructured and conventional plasma sprayed alumina-titania coatings. Surface \& Coatings Technology, 268 , 190-197.

[3] Kundera C., Kozior T. (2014), Research of the elastic properties of bellows made in SLS technology. Advanced Materials Research, 874, 77-81.

[4] Kanada T. (1997), Estimation of sphericity by means of statistical processing for roundness of spherical parts. „Precision Engineering”, Vol. 20 (2), pp. 117-122.

[5] Gleason E., Schwenke H. (1998), A spindless instrument for the roundness measurement of precision spheres. „Precision Engineering”, Vol. 22 (1), pp. 37-42.

[6] Głowacz A., (2014), Diagnostics of DC and Induction Motors Based on the Analysis of Acoustic Signals. Measurement Science Review, 14 (4), 257-262.

[7] Głowacz A., Głowacz Z., (2016), Diagnostics of stator faults of the single-phase induction motor using thermal images, MoASoS and selected classifiers. Measurement, 93, 86-93.

[8] Janecki D., Stępień K., Adamczak S. (2016) Sphericity measurements by the radial method: II. Experimental verification, Measurement Science and Technology, Vol. 27, 1, pp. 015006

[9] Ratajczyk E. (1968) Kontrola jakości powierzchni łożysk tocznych automatami AVIKO. „Mechanik”, Vol. 4/68, s. 203-207.

[10] Hagino T. et al., (2012), Sphericity Measurement Using Stitching Interferometry, Key Engineering Materials, Vols. 523-524, pp. 883-888.

[11] Guido Bartl et al., (2010), Interferometric determination of the topographies of absolute sphere radii using the sphere interferometer of PTB. "Meas. Sci. Technol”, Vol. 21, 115101 (8pp).

[12] Halkaci H.S., Mavi Ö., Yigit O., (2007) Evaluation of form error at semi-spherical tools by use of image processing. „Measurement”, Vol. 40 (9-10), pp. 860-867.

[13] Song L.M. et al., (2005) Novel 3D sphericity evaluation based on SFS-NDT. "NDT\&E International”, Vol. 38, pp. 442-447. 
[14] Stępień K. (2014), In situ measurement of cylindricity-Problems and solutions, Precision Engineering, Vol. 38 No. 3, pp. 697-701

[15] Janecki D., Stępień K., Adamczak S. (2016) Sphericity measurements by the radial method: I. Mathematical fundamentals, Measurement Science and Technology, Vol. 27, 1, pp. 015005

[16] Janecki D., Stępień K., Adamczak S. (2012), Problem of profile matching in sphericity mesurements by the radial method, Metrology and Measurement Systems, Vol. XIX, No. 4, pp. 703-714.

[17] Beranek, L.; Volf, L. \& Mikes, P. (2011). Coordinate Metrology Education Using Virtual CMM, Annals of DAAAM for $2011 \&$ Proceedings of the 22nd International DAAAM Symposium, 23-26th November 2011, Vienna, Austria, Volume 22, No. 1, ISSN 1726-9679, ISBN 978-3-901509-83-4, Katalinic, B. (Ed.), pp. 1323-1324, Published by DAAAM International Vienna, Vienna

[18] Runje, B. \& Medic, S. (2011). Measurement Uncertainty in Process of Line Scales Calibrating, Annals of DAAAM for 2011 \& Proceedings of the 22nd International DAAAM Symposium, 23-26th November 2011, Vienna, Austria, Volume 22, No. 1, ISSN 1726-9679, ISBN 978-3-901509-83-4, Katalinic, B. (Ed.), pp. 0093-0094, Published by DAAAM International Vienna, Vienna

[19] Poniatowska M. (2009), Research on spatial interrelations of geometric deviations determined in coordinate measurements of free-form surfaces. Metrology and Measurement Systems, 16 (3), 501-510. 